\title{
The Future of population health management: artificial intelligence as a cost-effective behavior change and chronic disease prevention and management solution
}

\begin{abstract}
Obesity and chronic diseases are pressing public health concerns, and population health management is one strategy to mitigate their consequences. Prevention and management are often possible through lifestyle changes, but the shortage of healthcare resources prevents large numbers of patients from receiving help in changing health behaviors. Artificial intelligence AI platforms have the potential to increase patients' access to care and deliver cost-effective, individualized care. Some interventions have already shown to be engaging to patients and effective for weight loss and other outcomes, and the future of AI in healthcare can include further research and specialized programs for a variety of chronic conditions.
\end{abstract}

Keywords: artificial intelligence, population health management, chronic disease, behavior change, obesity
Volume 6 Issue 5 - 2017

\author{
Natalie Stein \\ Department of Public Health, USA
}

Correspondence: Natalie Stein, Department of Public Health, College of Human Medicine, Michigan State University, 200 East Ist Street, Flint Campus, Michigan, 48502, USA, Tel I8I 0600560I, Email steinnal@msu.edu

Received: November 20, 2017 | Published: December 8, 2017
Abbreviations: AI, artificial intelligence; PHM, public health management; CBT, cognitive behavioral therapy

\section{Introduction}

\section{Chronic disease burden and modifiable contributing factors}

Chronic diseases are an immense public health concern in the United States. They are the leading causes of death and disability, and chronic and mental health conditions have an annual economic cost of nearly $\$ 2.5$ trillion. ${ }^{1}$ An estimated 157 million individuals are projected to have at least one chronic disease by $2020 .{ }^{2}$ Chronic conditions can largely be prevented and, once they do occur, managed to lower costs and reduce morbidity and mortality, through making healthier lifestyle choices. Maintaining a healthy weight, following recommendations for diet and physical activity, avoiding tobacco use and alcohol abuse, adhering to prescription medication instructions, and self-monitoring indicators, such as weight, blood pressure, and blood glucose, are all known to lower the burden of chronic disease, but most individuals fall short on one or more of these health behaviors. ${ }^{1}$

\section{Public health management and challenges in healthcare}

Public health management (PHM) is an approach in which healthcare organizations seek to "effectively manage the health of their total population, not just the patients who seek care". ${ }^{3}$ It is recognized as potentially effective for chronic disease prevention and care, but effective PHM faces multiple challenges, including a shortfall of healthcare resources. Chronic disease management is expensive, with a patient with 0,1 , or 2 chronic conditions averaging an annual cost of $\$ 1,177, \$ 2,915$, or $\$ 4,731$, respectively. ${ }^{4}$ Despite these high costs, patients have relatively little access to providers, with patients with 0,1 , or 2 chronic conditions making only 2.8 , 3.7 , or 4.9 annual clinician visits, respectively. So, few interactions with healthcare providers seem inadequate when recognizing the profound impact of daily health behaviors on chronic disease risk and management. Another challenge in managing population health is its overall lack of customization, since recommendations are developed for entire populations and may not be customized before being given to individual patients. A healthcare organization, for example, might provide all of its patients with diabetes an identical healthy living pamphlet with guidelines for nutrition, physical activity, and selfmonitoring without considering inter-patient variations such as dietary preferences or baseline physical activity levels. Further customization could consider intra-patient variations on a daily or even minute-tominute basis, with specialized counseling for situations such as when patients need encouragement to exercise, a reminder to measure blood glucose, or a suggestion for a healthy snack based on the time of day and what the patient has already eaten that day.

\section{Conclusion}

\section{Artificial intelligence for providing unlimited, personalized care}

This kind of intensive, personalized healthcare may seem unattainable with a traditional healthcare model because of costs and logistics such as paying medical professionals and scheduling appointments, but cost and logistical barriers can be lowered using technology such as artificial intelligence (AI), or the result is the ability to complement care offered by live healthcare providers. It can integrate broad strategies of PHM to reach many patients and promote population-based recommendations, while at the same time tailoring care to each individual patient, using a model of healthcare based on an "N of 1." For example, consistent with research and population guidelines, AI coaching might recommend breaks in sedentary behavior and controlled carbohydrate consumption for individuals with diabetes or pre-diabetes, and remind patients that 10-minute bouts of physical activity are beneficial. At the same time, the AI might assist the patient in setting physical activity goals based on the patient's baseline or current levels, rather than automatically setting 
a patient's activity goal at the national standard of 150 minutes per week of moderate-intensity activity.

Because an AI platform is able to gather so much data and "be with" the patient at any time, it can "learn" and respond to a patient's usual habits and health measures. Furthermore, it can detect and respond to deviations from the patient's norm, allowing it to, for example, praise the patient for eating more vegetables than usual the previous day, or ask the patient if she wants to get in a walk at lunchtime if she skips her usual morning walk, or guide the patient through stress management techniques if she reports The result is the ability of the AI to not only target each individual patient, but to further customize care to accommodate a single patient's own daily, hourly, or even minute-tominute variations. Multiple daily interactions with doctors or nurses are not feasible for the majority of patients, but the AI platform allows for unlimited coaching. In a Diabetes Prevention Program delivered via AI, patients at high risk for diabetes averaged 41 unique one-onone sessions with their AI provider in a 16-week program. This rate of interaction is over 40 times higher than the average of 3.7 clinic visits per year for an individual with a single chronic disease. ${ }^{4,5}$

\section{Measures of success with Al}

AI providers have already been linked to improved patient health behaviors and outcomes. In a 15-week study, users lost $2.38 \%$ of baseline body weight, which was comparable to the amount achieved in interventions that relied on in-person components. ${ }^{6}$ during the course of the study; users also increased their percentage of healthy meals logged by $31 \%$. The AI may have been successful due to its inclusion of best-practice elements for behavior change and chronic disease prevention and management programs. The AI incorporated cognitive behavioral therapy (CBT) strategies, targeted both diet and exercise as key behaviors for health, ${ }^{7}$ and was available to users $24 / 7 / 365$, as recommended for behavior change programs. ${ }^{2}$ Common barriers to multiple chronic disease management, such as lack of communication with members of the healthcare team, ${ }^{2}$ can also be addressed via AI platforms. For example, the AI provider could suggest that a patient contact her doctor if her blood pressure is out of range, and even alert a provider or connect a patient to a provider. The high rate of engagement of over 2.5 unique sessions per week, along with minimal associated cost is another promising sign for health outcomes and efficiency in the healthcare system, since increased engagement is known to reduce poor health outcomes. ${ }^{3}$

\section{Future directions for Al}

The healthcare system is resource constrained, and a successful future depends on cost-effective solutions. Digital interventions such as $\mathrm{AI}$ are infinitely scalable, and they can be effective in precipitating healthy behavior change and improve chronic disease prevention and management. Future studies should continue to investigate the effectiveness of AI in promoting behavior change in various populations and health contexts. Current and future AI programs can target a range of chronic conditions, including obesity, diabetes, hypertension, behavioral health, and arthritis, that respond to a patient's daily lifestyle choices and can be mitigated with unlimited, individualized care.

\section{Acknowledgement}

None.

\section{Conflict of Interest}

Natalie Stein consults with Lark Technologies.

\section{Funding}

None.

\section{References}

1. Centers for Disease Control and Prevention. USA: Chronic Diseases: The Leading Causes of Death and Disability in the United States. 2017.

2. Clarke JL, Bourn S, Skoufalos A, et al. An innovative approach to health care delivery for patients with chronic conditions. Popul Health Manag. 2017;20(1):23-30.

3. Hibbard JH, Greene J, Sacks RM, et al. Improving population health management strategies: identifying patients who are more likely to be users of avoidable costly care and those more likely to develop a new chronic disease. Health Serv Res. 2017;52(4):1297-1309.

4. Gerteis J, Izrael D, Deitz D, et al. Multiple chronic conditions chartbook: 2010 medical expenditure survey data. Agency for Healthcare Research and Quality, USA. 2014;1-52.

5. Stein N. User Engagement with an Artificial Intelligence Platform for Pre-Diabetes, USA. 2017.

6. Stein N, Brooks K. A fully automated conversational artificial intelligence for weight loss: longitudinal observational study among overweight and obese adults. JMIR Diabetes. 2017;2(2):e28.

7. Tkatch R, Musich S, MacLeod S, et al. Population health management for older Adults: Review of interventions for promoting successful aging across the health continuum. Gerontol Geriatr Med. 2016;2:2333721416667877. 